\title{
Idiopathic Intracranial Hypertension in a Prepubertal Pediatric Japanese Patient Complicated by Severe Papilledema
}

\author{
Akiko Oka Kengo Ikesugi Mineo Kondo \\ Department of Ophthalmology, Mie University Graduate School of Medicine, Tsu, Japan
}

\section{Key Words}

Idiopathic intracranial hypertension · Papilledema - Ventriculoperitoneal shunt

\begin{abstract}
Purpose: To report a rare case of idiopathic intracranial hypertension (IIH) in a prepubertal pediatric patient. Case Report: The patient was an 11-year-old Japanese boy. Initially, an ophthalmologist found severe papilledema, and the patient was diagnosed with IIH. He was unresponsive to conservative therapy, and a ventriculoperitoneal shunt was inserted in the Neurosurgery Department because the visual impairment was severe. Twelve months after the shunt, the improvement in vision was limited due to optic disc atrophy. Conclusion: Ophthalmologists need to be more aware of II, especially in cases with severe papilledema that can lead to permanent reduction of vision.

\section{Introduction}

Idiopathic intracranial hypertension (IIH) is characterized by increased intracranial pressure in the absence of an intracranial space-occupying lesion, cerebral ventricular enlargement, or abnormal increase in the cerebrospinal fluid. In general, IIH affects obese women but can occasionally occur in children. The clinical features of children with IIH are different from those of adults [1]. There are also regional differences in the prevalence of IIH; in Japan, IIH affects only 0.03 individuals per 100,000 when adults and children are included [2]. We report our findings in an 11-year-old Japanese boy with IIH complicated by severe papilledema.

\section{KARGER}

Kengo Ikesugi, MD

Department of Ophthalmology

Mie University Graduate School of Medicine

2-174 Edobashi, Tsu, Mie 514-8507 (Japan)

E-Mail ikesugi@clin.medic.mie-u.ac.jp 
Oka et al.: Idiopathic Intracranial Hypertension in a Prepubertal Pediatric Japanese Patient Complicated by Severe Papilledema

\section{Case Report}

The patient was an 11-year-old boy with no notable medical or family history. For about 3 months, he had occasional general malaise, headaches, and vomiting. The patient then began to complain of difficulty in turning his neck and was referred to an orthopedic clinic. No abnormalities were detected in the head and neck in the computed tomographic (CT) images and the magnetic resonance images (MRIs). In addition, all hematological tests were within the normal limits.

After 1 month, the patient had a transient reduction in vision, and when his vision worsened in both eyes, the patient was brought to an ophthalmologist. At the initial examination, his visual acuity was 20/200 in the right eye and 20/100 in the left eye. The intraocular pressure of the right and left eyes was 19 and $20 \mathrm{~mm} \mathrm{Hg}$, respectively. No clear differences in the pupillary light reflexes of the right and left eyes were found. A relative afferent pupillary defect was not present. The anterior segment of the eye was normal, but ophthalmoscopy showed a marked swelling of the optic disc and severe retinal exudates in both eyes (fig. 1).

The patient was admitted for comprehensive examinations on the same day. Physical and hematological abnormalities were not found. Head X-rays showed dehiscence of the coronal suture, which suggested intracranial hypertension. MRI showed no space-occupying lesions in the brain, but did show tortuous optic nerves, enlarged spinal fluid cavity, and flattened posterior sclera (fig. 2). MR venography did not show clear signs of a sinus thrombosis. Cerebrospinal tap demonstrated a marked increase in the cerebrospinal pressure with an opening pressure of 350-500 $\mathrm{mm} \mathrm{H}_{2} \mathrm{O}$, but the cerebrospinal fluid contents were normal.

Based on these findings, the patient was diagnosed with $\mathrm{IHH}$, and acetazolamide, concentrated glycerin, and steroid were injected intravenously. Initially, the intracranial pressure decreased after the treatment, but after 1 month, the intracranial hypertension and symptoms did not improved. Thus, a ventriculoperitoneal (VP) shunt was inserted in the Neurosurgery Department, and the intracranial pressure was lowered. The ophthalmological findings have been stable thereafter.

The changes in the fundus and visual field during the course of the disease process are shown in fig. 3. At present, about 1 year after the onset, the papilledema has improved but the vision is $20 / 320$ in the right eye and 20/2,000 in the left eye. The limited improvement of vision was most likely due to the optic nerve atrophy.

\section{Discussion}

In 1893, Quinke [3] reported a disease characterized by increased intracranial pressure in the absence of a space-occupying lesion in the brain, ventricular enlargement, and abnormalities of the cerebrospinal fluid. This disease was referred to as pseudotumor cerebri or benign intracranial hypertension. However, because it was determined that this disease can cause severe and permanent reduction of vision, the word 'benign' was deleted. Subsequently, it has been referred to as IIH.

The prevalence of IIH in the USA is about 1 per 100,000 , and it mainly affects adult women in their 20s and 30s [4,5]. Among morbidly obese women, the frequency of IIH is reported to be as high as 19.3 per 100,000. Regional differences in the frequency of IIH have been reported; the frequency of IIH in Africa is 2.2 per 100,000 , while in Japan it is only 0.03 per 100,000 . More than $60 \%$ of children with IIH are older than 10 years. Additionally, there is an identifiable cause of the intracranial hypertension in $84 \%$ of children younger than 6 years $[1,2,6]$. Thus, most cases of intracranial hypertension are not idiopathic in young chil- 
Oka et al.: Idiopathic Intracranial Hypertension in a Prepubertal Pediatric Japanese Patient Complicated by Severe Papilledema

dren, and it is generally accepted that the incidence of true prepubertal IIH is much rarer than adult IIH.

It has recently been reported that the clinical features of childhood IIH, in particular prepubertal IIH, differ from those of adult IIH $[1,2]$. The differences are the absence of differences in the sex distribution at the onset and the absence of a significant correlation with obesity. In addition, adult IIH patients often complain of headaches, have transient low vision, and tinnitus, while pediatric IIH patients often complain of restlessness, dizziness, and neck pain. However, adolescent IIH patients have clinical features similar to that of adult IIH. Currently, there is ongoing prospective study of the treatment of IIH in adults, but no data exist in children [1]. Most children respond to typical medical treatment such as intravenous carbonic anhydrase or steroids. Indication for surgical treatment include initial severe visual dysfunction or a worsening of the results of a visual test [6]. At the moment, because the etiology of IIH in children remains unclear, a specific treatment has not yet developed [1].

Cerebral venous sinus thrombosis (CVST) can be a cause of intracerebral hypertension. Although there are overlapping symptoms in CVST and IIH, the treatments are different. Thus, CVST must be ruled out in the diagnosis of IIH, but it is not possible to diagnose CVST based solely on head CT and MRI examinations. It is essential to perform MR venography in all patients with intracranial hypertension to make a diagnosis of CVST. The incidence of CVST is $9.4 \%$ in patients with IIH [7]. Other causes for intracerebral hypertension such as acute toxic encephalopathy and arachnoiditis are usually ruled out by MRI examination and medical history.

Our patient was not obese and was prepubertal. He did not have a history of head injury, medication use, or otitis media, and MRI and MRV ruled out cranial sinus thrombosis and other possible causes. These findings suggested that the patient had true IIH.

Several reports have discussed the prognosis for the visual function in pediatric patients with IIH. While some studies reported that visual impairments were extremely rare in these children, and when present, they were not severe or permanent. However, others found that 13-60\% had some degree of visual impairment, and 9\% had severe persistent impairments $[1,6,8]$. One study compared the visual impairments in IIH patients at different ages and reported that the prognosis for good visual function was worse in adolescent patients than in prepubertal patients [9]. Most studies found that pediatric IIH patients responded to therapy better than adult patients [8]. However, a surgical procedure was necessary in our case. Since visual impairment is the only severe complication for both children and adults, ophthalmologists can play an important role during the follow-up of this disease. It has been suggested that there is a correlation between poor visual prognosis and the presence of severe papilledema at the initial visit [10]. Surgery is necessary when the visual impairment is severe or intracranial pressure is unresponsive to conservative therapy $[1,10,11]$.

Our patient had severely reduced vision in both eyes at the initial visit, and because the vision did not improve after medical therapy, a VP shunt was created. Although the papilledema improved over time, the patient had residual optic nerve atrophy, and the visual acuity did not recover. In one report on children younger than 11 years of age, it was found that the visual function did not change markedly after therapy [12], while in another report, it was reported that the visual acuity transiently decreased to less than hand motion due to optic nerve atrophy, but finally improved to 20/20 [6]. This report indicates that our patient should be carefully monitored in the future.

In conclusion, we report our findings in a case of severe pediatric IIH which is very rare in the Japanese population. While many studies have found that the prognosis is generally good in pediatric IIH patients $[1,6,11]$, a collaboration among pediatricians, neurosurgeons, and ophthalmologists is essential for early diagnosis and treatment of IIH. The role of an 
Oka et al.: Idiopathic Intracranial Hypertension in a Prepubertal Pediatric Japanese Patient Complicated by Severe Papilledema

ophthalmologist is necessary for early detection, and the therapeutic planning to prevent vision reduction which is the only severe complication of IIH.

\section{Disclosure Statement}

Written informed consent was obtained from the patient for publication of this case report and any accompanying images.

\section{Statement of Ethics}

The authors declare that there are no conflicts of interest regarding the publication of this paper.

\section{References}

$\checkmark 1$ Ko MW, Liu GT: Pediatric idiopathic intracranial hypertension (pseudotumor cerebri). Horm Res Paediatr 2010;74:381-389.

-2 Yabe I, Moriwaka F, Notoya A, Ohtaki M, Tashiro K: Incidence of idiopathic intracranial hypertension in Hokkaido, the northern-most island of Japan. J Neurol 2000;247:473-475.

-3 Quincke H: Über Meningitis serosa und verwandte Zustände. Dtsch Z Nervenheilkd 1897;9:149-168.

-4 Kesler A, Bassan H: Pseudotumor cerebri - idiopathic intracranial hypertension in the pediatric population. Pediatric Endocrinol Rev 2006;3:387-392.

5 Ball AK, Clarke CE: Idiopathic intracranial hypertension. Lancet Neurol 2006;5:433-442.

6 Babikian P, Corbett J, Bell W: Idiopathic intracranial hypertension in children: the Iowa experience. J Child Neurol 1994;9:144-149.

7 Lin A, Foroozan R, Danesh-Meyer HV, De Salvo G, Savino PJ, Sergott RC: Occurrence of cerebral venous sinus thrombosis in patients with presumed idiopathic intracranial hypertension. Ophthalmology 2006;113:2281-2284.

8 Cinciripini GS, Donahue S, Borchert MS: Idiopathic intracranial hypertension in prepubertal pediatric patients: characteristics, treatment, and outcome. Am J Ophthalmol 1999;127:178-182.

19 Rogers DL: A review of pediatric idiopathic intracranial hypertension. Pediatr Clin North Am 2014;61:579590.

10 Stiebel-Kalish H, Kalish Y, Lusky M, Gaton DD, Ehrlich R, Shuper A: Puberty as a risk factor for less favorable visual outcome in idiopathic intracranial hypertension. Am J Ophthalmol 2006;142:279-283.

-11 Standridge SM: Idiopathic intracranial hypertension in children: a review and algorithm. Pediatr Neurol 2010;43:377-390.

$>12$ Stiebel-Kalish H, Lusky M, Yassur Y, Kalish Y, Shuper A, Erlich R, Lubman S, Snir M: Swedish interactive thresholding algorithm fast for following visual fields in prepubertal idiopathic intracranial hypertension. Ophthalmology 2004;111:1673-1675. 
Oka et al.: Idiopathic Intracranial Hypertension in a Prepubertal Pediatric Japanese Patient Complicated by Severe Papilledema
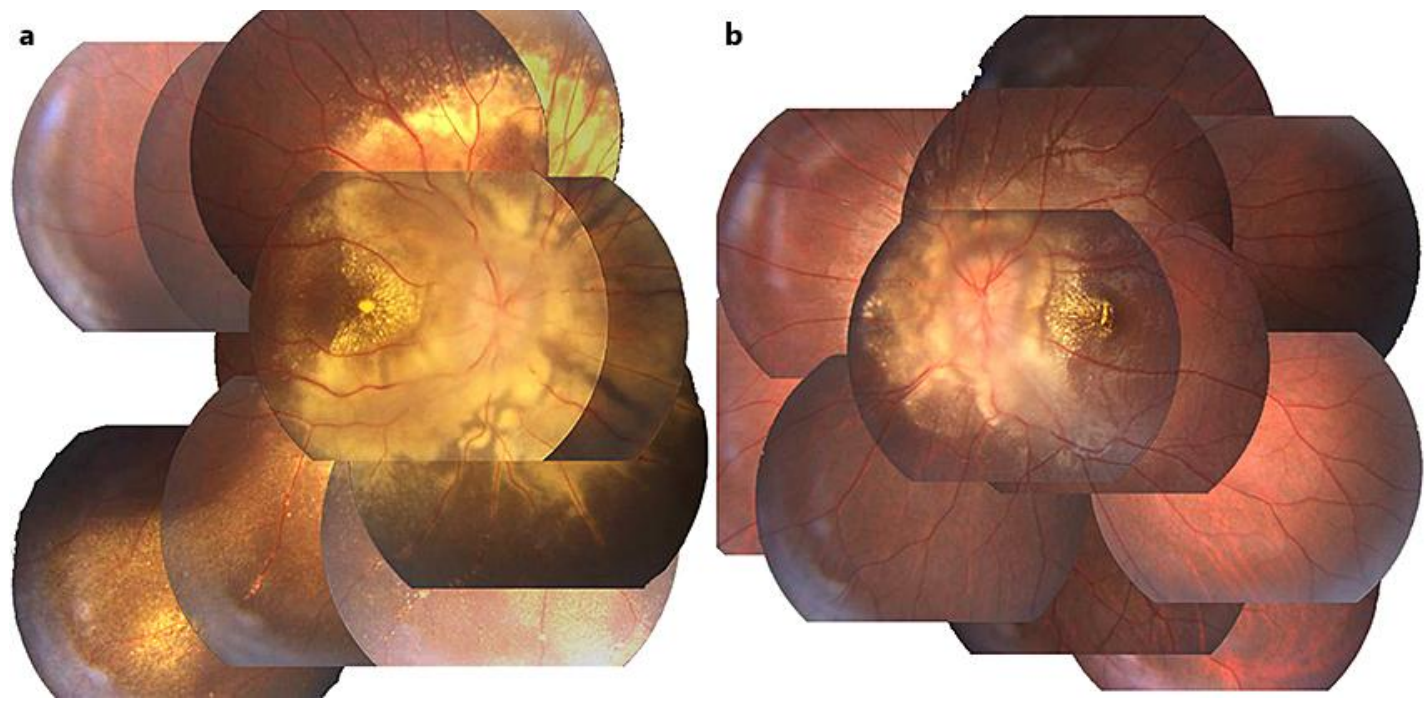

Fig. 1. Fundus photographs at the initial visit showing severe papilledema in both eyes of an 11-year-old patient with idiopathic intracranial hypertension. a Right eye. b Left eye.
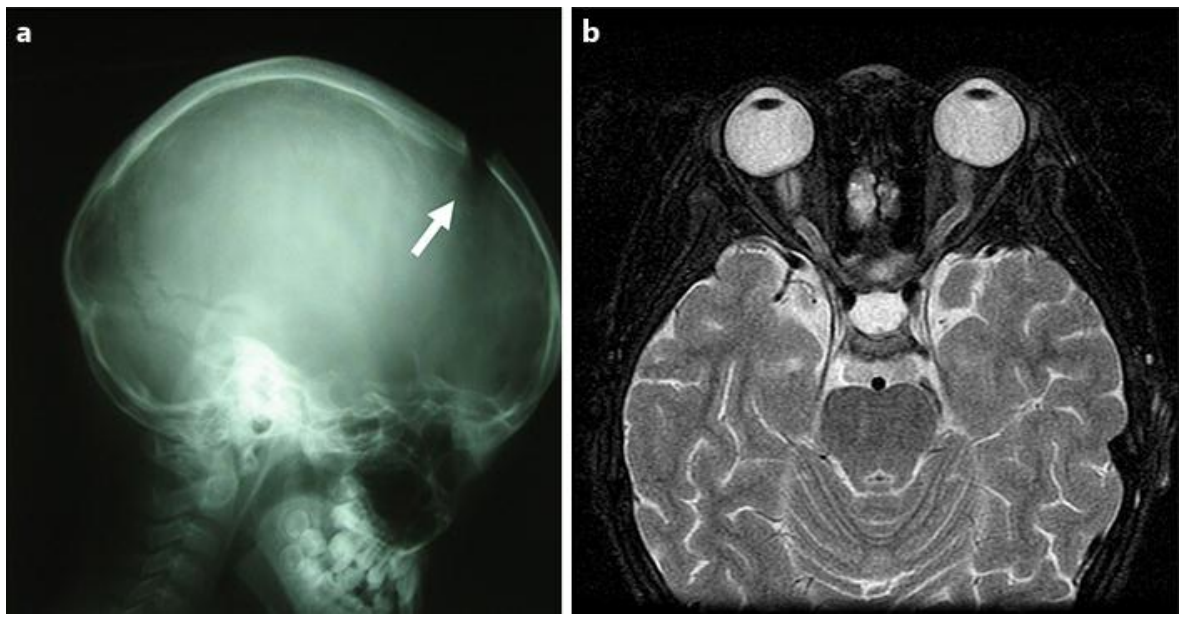

Fig. 2. Head X-ray and MRIs of the young boy described in fig. 1. a Head X-ray showing dehiscence of the coronary suture. $\mathbf{b}$ Head MRI shows flattening of the posterior sclera and tortuous optic nerve in both eyes. 
Case Reports in

Ophthalmology
Case Rep Ophthalmol 2016;7:148-153

DOI: 10.1159/000443951

(C) 2016 The Author(s). Published by S. Karger AG, Basel www.karger.com/cop

Oka et al.: Idiopathic Intracranial Hypertension in a Prepubertal Pediatric Japanese Patient Complicated by Severe Papilledema
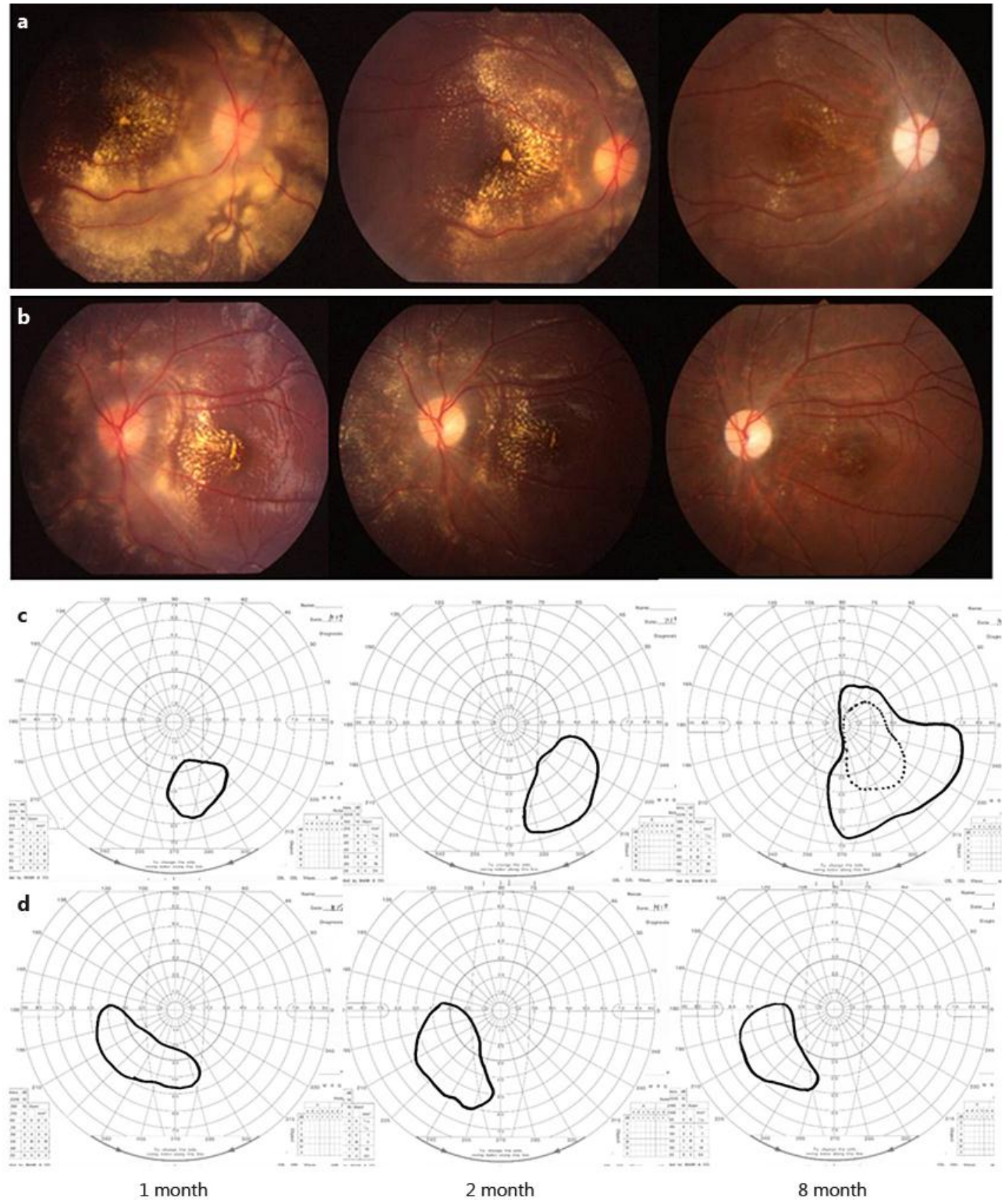

Fig. 3. Chronological changes in the optic disc and visual field. Papilledema of both eyes is resolved. The visual field of the right eye is gradually improved. a, c Right eye. $\mathbf{b}, \mathbf{d}$ Left eye. 